\title{
The Institute of Mathematical Sciences (IMS) at the Chinese University of Hong Kong
}

\section{by Zhouping Xin}

\author{
Department of Mathematics, The Chinese University of Hong Kong
}

Under the leadership of Professor Shing-Tung Yau, The Institute of Mathematical Sciences (IMS) at The Chinese University of Hong Kong has developed steady fastly and become one of leading centers internationally in mathematical researches both in theory and applications since its establishment in 1993. The IMS engages in in-depth and interdisciplinary researches in geometry and mathematical physics, partial differential equations and fluid mechanics, number theory and representation theory, topology, theoretical computer science, numerical analysis and large scale computations, and dynamical systems and optimizations, etc. The emphasizes of the IMS are both in high quality researches in pure and applied mathematics and nurturing young scientists. A full

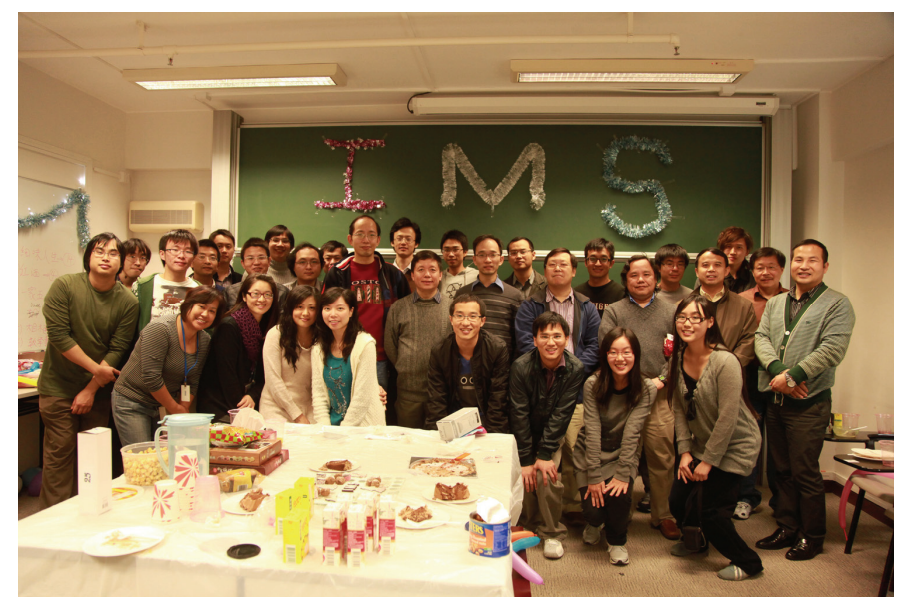

IMS Christmas party, 2011

range of post-graduate (in $\mathrm{PhDs}$ and MPhils), post-doctorial and visiting scholar programs are established. Conferences, workshops and lecture series are regularly organized. The IMS has also built up strong connections with leading universities and research centers in China and abroad. It also manages the publication of three international research journals: Methods and Applications of Analysis (MAA), The Asian Journal of Mathematics (AJM), and Communications in Information and Systems (CIS).
Currently, the IMS has ten permanent members which include one Fields Medalist and Wolf Prize Laureate (Prof. Shing-Tung Yau), one Turing Medalist (Prof. Andrew Chi-Chih Yao), one Morningside Gold Medalist (Prof. Zhouping Xin), two Morningside Silver Medalists (Prof. Hon Fu Chan, Raymond and Prof. Juncheng Wei), and two Chern Prize Winners (Prof. Nai-Chung Leung, Conan and Prof. Jiu-Kang Yu). Meanwhile, it has five postdoctoral fellows, 20 graduate students, and around 70 academic visitors (per year) at this moment. The IMS has trained many excellent $\mathrm{PhDs}$ and postdoctoral fellows who has become distinguish researchers in their field. The research strength of the IMS has further enhanced by the joining of Prof. Jiu-Kang Yu and Prof. Yi-Jen Lee last fall both from Purdue University. Prof. Yu is a leading expert in number theory and representation theory. He was awarded the Chern Prize for his foundation work on the construction of supercuspidal representations. His recent work solved long standing problems in Vinberg theory and Moy-Prasad theory. He has also published significant works in algebraic geometry and differential geometry. Prof. Yu was a Sloan Fellow, a Purdue University Faculty Scholar, and a Fellow of the AMS. The research interests of Prof. Yi-Jen Lee lie in geometry and topology, in particular, gauge theory and symplectic geometry. Her recent work focuses on relating some major invariants from either fields. Both Prof. Yu and Prof. Lee got their PhDs from Harvard University. 